Original article

\author{
New York State \\ Department of Health, \\ Albany, New York, \\ USA \\ J Mikl \\ P F Smith
}

School of Public

Health University at

Albany, State

University of New

York, Albany, NY, USA

Z Sudar

P F Smith

National Reference

Laboratory for AIDS,

Prague, Czech

Republic

M Bruckova

J Jedlicka

Apolinar Clinic,

Prague, Czech

Republic

V Kastankova

R Tryzna

State University of

New York, Brooklyn,

NY, USA

J DeHovitz

Correspondence to:

Jaromir Mikl, New York

State Department of Health,

Bureau of HIV/AIDS

Epidemiology, ESP, Corning

Tower, Room 772, Albany,

NY, 12237 USA.

Accepted for publication

9 October 1997

\title{
HIV infection and high risk behaviour among patients attending an STD referral clinic in Prague, Czech Republic
}

\author{
J Mikl, Z Sudar, P F Smith, M Bruckova, J Jedlicka, V Kastankova, R Tryzna, J DeHovitz
}

Objective: This survey was conducted to determine the prevalence of HIV infection and associated risk behaviour in a high risk population of clients attending an STD clinic in Prague, Czech Republic.

Methods: Between September 1994 and January 1995 clients entering the Apolinar STD clinic in Prague, Czech Republic, were enrolled in a blinded, unlinked HIV antibody seroprevalence study. Non-identifying demographic characteristics, STD diagnoses, HIV risk exposures, and voluntary HIV testing experience were extracted from medical charts.

Results: Of 1394 patients for whom serum was available for testing, one was positive for HIV (HIV prevalence $0.07 \%, 95 \%$ confidence interval (CI) $0.01,0.41 \%$ ). This person was one of 28 men having sex with men (MSWM) (HIV prevalence among MSWM 3.6\%, 95\% CI 0.6, $17.7 \%$ ). Among the 775 male clients, $75.5 \%$ had heterosexual unprotected sex, $11.1 \%$ had sex with high risk partners or prostitutes, $3.6 \%$ were MSWM, $1.0 \%$ were injecting drug users (IDUs), $0.7 \%$ were both MSWM and IDUs, and $6.8 \%$ and $1.8 \%$ had other or no recognised risk for HIV/STDs, respectively. Among the 619 female clients, $74.5 \%$ had heterosexual unprotected sex, $11.6 \%$ were prostitutes, $7.8 \%$ had sex with high risk partners, $1.1 \%$ were IDUs, and $3.9 \%$ and $2.3 \%$ had other or no recognised risk, respectively. The 304 adolescent patients (age 11-19 years) differed significantly $(\mathrm{p}<0.05)$ in risk behaviour and STD diagnoses from the 1090 patients who were 20 years and older. Adolescents were significantly more likely to be female $(58.6 \% v 40.5 \%, \mathrm{OR}=2.1)$, IDUs $(3.6 \% v 0.4 \%, \mathrm{OR}=10.2)$, prostitutes $(8.9 \% v 4.7 \%, \mathrm{OR}=2.0)$, and have sex partners with STDs $(7.6 \% v 4.4 \%, \mathrm{OR}=1.8)$. The adolescent patients were also significantly more likely to be diagnosed with gonorrhoea $(21.1 \% v 12.3 \%, \mathrm{OR}=1.9)$ and non-gonococcal urethritis $(27.6 \% v 17.2 \%, \mathrm{OR}=1.8)$, and significantly less likely to have been tested previously for $\mathrm{HIV}(19.1 \%$ v $31.9 \%$, OR=0.5).

Conclusions: HIV infection is currently uncommon in this population. However, the high rates of unprotected sex, prostitution, injecting drug use, and STDs, especially among adolescents, provide the basis for an epidemic in this population. Aggressive prevention education should be started before adolescence.

(Sex Transm Inf 1998;74:128-130)

Keywords: epidemiology; HIV-1 infection; STDs; risk factors; adolescents; sexual transmission; Czech Republic

\section{Introduction}

Epidemics of HIV and sexually transmitted diseases have historically been associated with social and political change, especially drug use, poverty, and travel. In the Czech Republic major sociopolitical changes have occurred since 1989. Byproducts of these changes have included increases in commercial sex, drug use, and sexually transmitted diseases. ${ }^{12}$

In the Czech Republic, the first case of AIDS was diagnosed in $1984,{ }^{3}$ and the first case of laboratory documented HIV was diagnosed in 1985. ${ }^{4}$ By the end of February 1996, 259 HIV cases had been diagnosed. As in other early European HIV/AIDS epidemics, the majority $(54 \%)$ of the cases were homosexual/bisexual men, and $22 \%$ were acquired through heterosexual contact. Only 3\% were injecting drug users (IDUs). Twenty three (9\%) of the HIV positive cases were adolescents (10-19 years). Since 1993, the proportion of heterosexually transmitted HIV cases has increased nearly twofold, while the proportion among homosexual men has declined. The emergence of this pattern poses particular risk for patients with sexually transmitted diseases, who are at both biological and behavioural risk for HIV infection.

We performed a cross sectional study of clients entering an STD referral clinic in Prague, Czech Republic between September 1994 and January 1995. The objectives were: to determine the seroprevalence of HIV antibodies in this population; to determine demographic characteristics of the clients including age, sex, ethnicity, and residence; to determine the prevalence of HIV risk behaviour and STDs in this population; and to ascertain the acceptability of voluntary HIV testing among these patients.

\section{Materials and methods}

Between September 1994 and January 1995, 1500 consecutive clients entering the Apolinar STD clinic in Prague, Czech Republic, were enrolled in a blinded, unlinked HIV antibody seroprevalence study. Non-identifying demographic characteristics, STD diagnoses, HIV risk exposures, and voluntary HIV testing 
Table 1 Characteristics of clients entering an STD clinic by age category. Prague, Czech Republic, 1994-5

\begin{tabular}{|c|c|c|c|c|}
\hline \multirow[b]{2}{*}{ Characteristic } & \multicolumn{2}{|c|}{$11-19$ years } & \multicolumn{2}{|c|}{$20+$ years } \\
\hline & No & $\%$ & No & $\%$ \\
\hline \multicolumn{5}{|l|}{ Sex: } \\
\hline Male & 126 & 41.4 & 649 & 59.5 \\
\hline Female & 178 & 58.6 & 441 & 40.5 \\
\hline \multicolumn{5}{|l|}{ Risk factors for HIV infection ${ }^{\star}$ : } \\
\hline MSWM & 4 & 1.3 & 24 & 2.2 \\
\hline IDUs & 11 & 3.6 & 4 & 0.4 \\
\hline MSWM and IDU & 1 & 0.3 & 4 & 0.4 \\
\hline Prostitution & 27 & 8.9 & 51 & 4.7 \\
\hline Men having sex with prostitute & 3 & 1.0 & 46 & 4.2 \\
\hline Sex partner IDU & 6 & 2.0 & 8 & 0.7 \\
\hline Sex partner with STD & 23 & 7.6 & 48 & 4.4 \\
\hline Unprotected penile-vaginal & 205 & 67.4 & 841 & 77.2 \\
\hline Othert & 24 & 7.9 & 53 & 4.9 \\
\hline None reported & 6 & 2.0 & 22 & 2.0 \\
\hline \multicolumn{5}{|l|}{ STD diagnoses $\star:$} \\
\hline Serological diagnosis of syphilis & 3 & 1.0 & 27 & 2.5 \\
\hline Neisseria gonorrhoeae & 64 & 21.1 & 134 & 12.3 \\
\hline Chlamydia trachomatis & 84 & 27.6 & 188 & 17.2 \\
\hline Genital herpes & 4 & 1.3 & 20 & 1.8 \\
\hline Other genital ulcers & 5 & 1.6 & 34 & 3.1 \\
\hline Other $\neq$ & 141 & 46.4 & 612 & 56.1 \\
\hline None & 42 & 13.8 & 144 & 13.2 \\
\hline \multicolumn{5}{|l|}{ Acceptance of HIV testing: } \\
\hline Before and during the study & 56 & 18.4 & 333 & 30.6 \\
\hline Only during the study period & 240 & 78.9 & 717 & 65.8 \\
\hline Only before the study period & 2 & 0.7 & 14 & 1.3 \\
\hline Never tested & 6 & 2.0 & 26 & 2.4 \\
\hline Total & 304 & 100.0 & 1090 & 100.0 \\
\hline
\end{tabular}

MSWM = men having sex with men; IDUs = injecting drug users

*Indicates prevalence of HIV risk factors and STD diagnoses. The number and percentages do not add up to the total number of clients and $100 \%$, respectively, owing to multiple HIV risks and STD diagnoses.

†Includes patients self reporting promiscuity, transfusion recipients, rape victims, and individuals with tattoos.

‡Includes patients diagnosed with genital warts, pediculosis pubis, candidiasis, mycoplasma ureaplasma, and Gardnerella vaginalis infection.

experience were extracted from patients' records.

Syphilis serological status was determined using the VDRL test, and for confirmation either the TPHA or FTA-ABS was used. The Gen-Probe Pace 2 system (Gen-Probe Inc, San Diego, CA, USA), a rapid DNA probe test using nucleic acid hybridisation was used to detect both Neisseria gonorrhoeae and Chlamydia trachomatis from female endocervical and male urethral swab specimens.

An aliquot of excess serum, derived from routine syphilis screening, was used for HIV testing. Serum samples were screened for antibody to HIV (type 1 and type 2) using an enzyme immunoassay (Fujirebio Inc, Tokyo, Japan). Replicate positive samples were tested by western blot assay (DuPont Pharmaceuticals, USA). A positive western blot was one showing the presence of any two of the p24, gp41, or gp120/160 bands; a negative western blot was showing no bands. Concurrent with their examination, all participating clients were offered an opportunity to consent to separate voluntary HIV testing.

We compared HIV risk, STD diagnoses, and acceptance of voluntary HIV testing for adolescent clients (11-19 years) with their older counterparts (20+ years). $\chi^{2}$ analyses were used to compare group differences using SAS software package (Cary, NC, version 6.03). Odds ratios and corresponding 95\% confidence intervals on factors associated with age were calculated using TRUE EPISTAT (Richardson, $\mathrm{TX}$, version 5.0).
This study was reviewed and approved by institutional review boards at the National Institute of Public Health in the Czech Republic, the State University of New York Health Science Center at Brooklyn, and the New York State Department of Health in Albany, New York, United States.

\section{Results}

Of the 1500 patients enrolled in the study, 106 patients were excluded from analysis because of lack of available serum for HIV testing. These patients were similar to the 1394 patients with serum, except that they were significantly more likely to be male $(79.2 \% v$ $55.6 \%$ ), to be tested for HIV previously $(69.8 \%$ v $29.1 \%)$, and were less likely to have Chlamydia trachomatis infection $(8.5 \% \quad v$ $19.5 \%$ ) (data not shown).

DEMOGRAPHICS

During the study period, 775 males and 619 females were evaluated (table 1 ). The mean age of patients was 26.2 years (range 11-77 years); the majority of them were 20 years and older, Czech nationals $(93.9 \%)$, and residents of Prague $(81.7 \%)$ (data not shown).

\section{HIV INFECTION PREVALENCE}

Of the 1394 patients with HIV test results, one tested positive for HIV. The HIV prevalence was $\quad 0.07 \% \quad(95 \%$ confidence interval $(C I)=0.01,0.41)$. This person was one of 28 men having sex with men (MSWM); thus, the HIV infection rate among MSWM was $3.6 \%$ (95\% CI=0.6, 17.7). The HIV positive person was 20-24 years of age from Prague, who was seen for treatment of gonorrhoea. He had had five sexual partners in the previous 12 months. During his examination he agreed to confidential, voluntary HIV testing. He reported being tested previously, outside of the study, for HIV antibody in June 1994 with a negative result.

RISK BEHAVIOUR, STD DIAGNOSES, AND

ACCEPTANCE OF HIV TESTING

In the overall sample $(n=1394), 75.0 \%$ of the patients reported heterosexual unprotected sex, predisposing them to potential STD and HIV infections. Among the female clients, $11.6 \%$ self identified as prostitutes and $7.3 \%$ reported having a sexual partner with an STD. Among the participating male patients, $6.3 \%$ reported to be clients of prostitutes, $3.6 \%$ reported having sex with men, and $1.4 \%$ had sexual partners who were IDUs. The most commonly diagnosed STD was $C$ trachomatis infection (19.5\%), followed by gonorrhoea $(14.2 \%)$. High acceptance $(96.6 \%)$ of confidential, voluntary HIV screening was observed (data not shown).

\section{AGE GROUP DIFFERENCES}

The 304 adolescent patients (11-19 years of age) differed significantly from the 1090 patients who were $20+$ years of age. The adolescents were significantly more likely to be female $(58.6 \%$ v $40.5 \%, \quad \mathrm{OR}=2.1)$, IDUs $(3.6 \%$ v $0.4 \%, \mathrm{OR}=10.2)$, prostitutes $(8.9 \% v$ $4.7 \%, \mathrm{OR}=2.0)$, to have a sex partner with an 
Table 2 Bivariate analysis of factors associated with younger age among clients entering an STD clinic. Prague, Czech Republic, 1994-5

\begin{tabular}{lllll}
\hline Characteristic & $\begin{array}{l}11-19 \text { years } \\
(n=304)\end{array}$ & $\begin{array}{l}20+\text { years } \\
(n=1090)\end{array}$ & $\begin{array}{l}\text { Odds } \\
\text { ratios }\end{array}$ & $95 \% C I$ \\
\hline Female sex & 178 & 441 & 2.1 & $1.6-2.7$ \\
Injecting drug use & 11 & 4 & 10.2 & $3.0-38.2$ \\
Prostitution & 27 & 51 & 2.0 & $1.2-3.3$ \\
Sex partner with STD & 23 & 48 & 1.8 & $1.0-3.1$ \\
$N$ gonorrhoeae infection & 64 & 134 & 1.9 & $1.3-2.7$ \\
$C$ trachomatis infection & 84 & 188 & 1.8 & $1.3-2.5$ \\
Prior HIV testing & 58 & 347 & 0.5 & $0.4-0.7$ \\
\hline
\end{tabular}

STD $(7.6 \%$ v $4.4 \%, \mathrm{OR}=1.8)$, and to be diagnosed with gonorrhoea $(21.1 \% \quad v 12.3 \%$, $\mathrm{OR}=1.9)$ and $C$ trachomatis infection $(27.6 \% v$ $17.2 \%, \mathrm{OR}=1.8$ ). Adolescents were also significantly less likely to have been tested previously for HIV $(19.1 \% v 31.9 \%$, OR=0.5) (tables 1 and 2).

\section{Discussion}

STD clinics are important sentinel sites for the surveillance of HIV infection because they serve people with sexual and often other risks, such as injecting drug use. ${ }^{5}$ HIV seroprevalence in this high risk population was low, $0.07 \%$. This is comparable with a previous 1991 study in which none of 245 patients tested was positive. ${ }^{6}$ The high acceptance rate $(96.6 \%)$ of confidential, voluntary HIV testing in this study is similar to that found in earlier surveys ${ }^{6}$ performed in the Czech Republic.

Adolescent patients were more likely to be female, IDUs, prostitutes, and to have a sex partner with STDs. These findings may be because females become sexually active and engage in high risk behaviour at an earlier age than males. ${ }^{7}$ We also found that adolescent patients were more likely to have $C$ trachomatis and $N$ gonorrhoeae infections than their older counterparts. These findings are consistent with the typical epidemiological pattern of these infections. ${ }^{8-10}$ The high gonorrhoea and chlamydia rates in the adolescent clients $(21.1 \%$ and $27.6 \%$, respectively) are troubling because of the potential consequences, such as pelvic inflammatory disease and facilitation of HIV transmission. Further, while the incidence of $N$ gonorrhoeae has been declining in the industrialised countries of western Europe since 1986 and in the United States since $1975,{ }^{11-15}$ it has been increasing in the former communist countries in eastern Europe. ${ }^{12}{ }^{16} C$ trachomatis is the most prevalent STD throughout Europe and the United States and its incidence is increasing throughout the world. ${ }^{11-15}$

The adolescents in our study were less likely to have been previously tested for HIV. We speculate that this is a function of age (less time for opportunity to be tested during their lives), but it might also indicate that they do not perceive themselves to be at high risk for HIV infection owing to lack of sufficient HIV related information.

Although blinded, unlinked surveys provide excellent estimates of HIV prevalence, our survey has limitations. Only one clinic was surveyed, thus it may not be representative of all STD clinics in the capital region of the Czech Republic; nor are the patients attending this clinic representative of all those with STDs (for example, those seen by private physicians) in this region. Secondly, we had access only to information that was routinely collected by the clinics and available in the medical records. Finally, the completeness of risk behaviour information collected may have varied among physicians.

The political and subsequent socioeconomic changes in the Czech Republic since 1989 have resulted in increased travel, migration, and economic dislocation. This has led to dramatic increases in commercial sex and presumably is contributing to the rise in STDs seen throughout the region. The Czech health care system has also been transforming from a state owned service to individual (private) practitioners resulting in a potential decrease in STD reporting as well as partner notification. In response to the growing problem of STDs and HIV there has been an increase in preventive efforts within the educational curriculum. At the same time, Czech television and radio increasingly began to provide information to the country on both epidemics. Evaluation programmes need to be developed to assess their impact.

In conclusion, although HIV infection is currently uncommon in this population, unprotected sex, prostitution, injecting drug use, and STDs, especially among adolescents, provide a substrate for a potential dramatic increase in HIV infection. Aggressive steps should be taken to lessen the chance of HIV infection in today's adolescents and tomorrow's future adolescents.

This study was funded in part by grant No 3 D43 TW00233 from the Fogarty International Center, National Institutes of Health, to State University of New York Health Science Center at Brooklyn.

1 Kastankova V. Increasing sexually transmitted disease rate among prostitutes in the Czech Republic. 7 Community Health 1995;20:219-12.

2 Netzelmann R. Patterns of prostitution in border areas. WHO report of a short-term assessment of the situation in the border region of the Czech Republic 1995. Geneva: WHO, Rorder region of the Czech Republic

3 Svejda J, Dlouhy P. Regional AIDS center activities in the Czech Republic. $\mathscr{f}$ Community Health 1995;20:197-201.

Czech Republic. F Community Health 1995;20:197-201.
Bruckova M, Jedlicka J. HIV/AIDS in the Czech Republic. Bruckova M, Jedlicka J. HIV/AIDS in
Community Health 1995;20:83-6.

5 Onorato IM, McCray E, Pappaioanou M, et al. HIV seroprevalence surveys in sexually transmitted disease clinics. Public Health Reports 1990;105:119-24.

6 The European Study Group. European community concerted action on HIV seroprevalence among sexually transmitted disease patients in 18 European sentinel networks. AIDS 1993;7:393-400.

7 D'Angelo LJ, Getson PR, Luban NLC, et al. Human immunodeficiency virus infection in urban adolescents: can we predict who is at risk? Pediatrics 1991;88:982-6.

8 Kohl PK. Epidemiology of sexually transmitted diseaseswhat does it tell us? Sex Transm Dis (Suppl) 1994;S81-3.

9 De Schryver A, Meheus A. Epidemiology of sexually transmitted diseases: the global picture. Bull World Health Organ 1990;68:639-54.

10 Rosenfeld WD. Sexually transmitted diseases in adolescents: update 1991. Pediatric Annals 1991;20:30312 .

11 Piot $\mathrm{P}$, Islam MQ. Sexually transmitted diseases in the 1990s. Global epidemiology and challenges for control Sexy Transm Dis (Suppl) 1994;S7-13.

12 Coutinho RA. Epidemiology of sexually transmitted diseases. Sex Transm Dis (Suppl) 1994;S51-2.

13 Meyer L, Goulet V, Massari V, et al. Surveillance of sexually transmitted diseases in France: recent trends and incidence. Genitourin Med 1994;70:15-21.

14 Suligoi B, Giuliani M, Binkin N, et al. The national surveillance system for sexually transmitted diseases in Italy.CDC lance system for sexually transmitted diseases in Italy.

15 Division of STD/HIV Prevention. Sexually transmitted disease surveillance, 1993. US Dept of Health and Human ease surveillance, 1993. US Dept of

16 Linglof T. Rapid increase of syphilis and gonorrhea in parts of the former USSR. Sex Transm Dis 1995;22:160-1. 